\title{
ON THE POSSIBILITY OF OBSERVING RADIO EMISSION FROM FLARE STARS
}

\author{
E. SCHATZMAN \\ Institut d'Astrophysique and Faculté des Sciences de Paris, France
}

Strong radio emissions are associated with solar flares. It seems worth while, therefore, to examine whether radio emission associated with flares and eruptions in nonstable stars could be observed. The first problem in this respect is establishing the threshold of sensitivity necessary to receive anything during an eruption or a flare; the second one is naturally fixing the duration of the radio emission, which can be much shorter in a given wavelength than the optical emission.

Let us first consider the classes of nonstable stars that have to be considered:

(a) T Tauri stars. According to the classification of Herbig [1] and Joy [2], we shall consider as $T$ Tauri stars, the stars of spectral classes $F$ to $G$, presenting a characteristic emission spectrum of large and irregular light variations with an amplitude of about 3 magnitudes.

(b) UV Ceti stars. These stars present, according to Haro [3], short-lived and nonperiodic outbursts lasting from a few minutes to approximately two hours; their spectral type ranges from dK6 down to late dM. But during the outburst the spectrum is much like the spectrum of some T Tauri stars. Consequently it seems right to suppose that UV Ceti stars merely form a prolongation of the class of $\mathrm{T}$ Tauri stars toward the late spectral types.

Two other classes of nonstable stars might be of interest, with respect to the problem of radio emission, e.g. SS Cygni stars and the novae. These stars raise a problem somewhat different from the problem of flare stars.

We shall estimate the amount of radio emission in flare stars according to two different methods.

(1) Let us suppose first that the stellar flares and the solar flares are of the same kind. Let us take as an example a type II burst [4] with a flux density at $100 \mathrm{Mc} / \mathrm{s}$ reaching $1^{-17}$ watts $(\mathrm{c} / \mathrm{s})^{-1}$ in a few minutes, and associated with a total emission of light of about $6 \times 10^{26} \mathrm{ergs} / \mathrm{second}$. An emission of $6 \times 10^{26} \mathrm{ergs} / \mathrm{second}$ corresponds to an absolute magnitude 21.5 , but is observed as a star of magnitude - 5.3. If we limit the sensitivity to $5 \times 10^{-25}$ watts $\mathrm{m}^{-2}(\mathrm{c} / \mathrm{s})^{-1}$, we find that a star reaching magnitude 14.6 during a flare could be observed on $100 \mathrm{Mc} / \mathrm{s}$.

(2) From recent experiments [5], it appears that the energy radiated as radiowaves from shock fronts amounts to about $10^{-5}$ to $10^{-6}$ times the shock's total energy. A shock's total internal energy, which is finally dissipated, is of the order of its mechanical energy. Therefore, the radio emission from 
a shockwave is also of the order of $10^{-5}$ to $10^{-6}$ times the energy radiated in visible and ultraviolet light. This order of magnitude is correct if one considers a type II burst; consequently, the results of the experiments on shockwaves and the extrapolation of the observations of solar flares lead to the same results.

We refer to three lists of variable stars: the list of Joy [2], the classification of RA Aurigae stars by Hoffmeister [6], and the list of Me stars (flare stars) compiled by P. E. Roques [7]. From the limit given, it can be seen that, at least in principle, about one-dozen stars may be expected to reach $5 \times 10^{-23}$ watts $\mathrm{m}^{-2}(\mathrm{c} / \mathrm{s})^{-1}$ during a flare, which seems well above the limit of sensitivity.

Let us consider now the duration of a type II burst in a flare star. As we know from the solar burst, the duration of the radio emission is very short, about five minutes. If we consider that such an emission is associated with plasma oscillations, we have to suppose that the emission in a certain frequency lasts as long as the plasma frequency has the same value. Therefore, the duration of the radio emission cannot be much longer than it is on the sun.

\section{REFERENCES}

[1] Herbig, G. H. Non-Stable Stars (I.A.U. Symposium No. 3). Cambridge, England, 1957.

[2] Joy, A. H. Ap. J. 102, 168, 1945.

[3] Haro, G. Non-Stable Stars (I.A.U. Symposium No. 3).

[4] Coutrez, R. Radio Astronomie. Uccle (Observatoire Royal de Belgique, Monographie No. 5), 1956.

[5] Gallet, R. Lecture given at Varenna, 1958.

[6] Hoffmeister, C. Astr. Nachr. 278, 24, 1949.

[7] Roques, P. E. P.A.S.P. 67, 34, 1955.

\section{Discussion}

Ryle: I should like to ask Dr. Schatzman what fraction of time we might expect to have this emission. We have made a comparison of our radio-star records taken at different times over a period of eight years in an attempt to detect any variations in the intrinsic radio luminosity of any of the sources, and we might therefore be able to use this analysis to fix an upper limit to the intensity of the type of emission now suggested. If the flare stars produced an emission lasting for several minutes and occurring fairly frequently, we should have been able to detect it if the flux exceeded about $5 \times 10^{-25}$ watts $\mathrm{m}^{-2}(\mathrm{c} / \mathrm{s})^{-1}$ at a frequency of $81.5 \mathrm{Mc} / \mathrm{s}$.

Schatzman: I would say that the duration of the radio emission at a given wavelength is much shorter than the duration of the optical emission. In a T Tauri star, where the eruption occurs with a frequency of once in five to ten days, and lasts a few hours, I would suggest that the radio emission lasts a few minutes. Some flare stars flare very frequently, e.g. every hour. 